\title{
Microencapsulación de células y tejido para terapia celular*
}

\author{
Dr. PATRICIO CABANÉ T. ${ }^{1}$, Int. ANDRÉS ALVO V. ${ }^{4}$, \\ Drs. ANDRÓNICO NEIRA-CARRILLO ${ }^{2}$, PABLO CAVIEDES F. ${ }^{3}$, PATRICIO GAC E. ${ }^{1}$ \\ 1 Departamento de Cirugía, Hospital Clínico Universidad de Chile. \\ 2 Facultad de Ciencias Veterinarias y Pecuarias Universidad de Chile. \\ 3 Facultad de Medicina, Universidad de Chile. \\ ${ }^{4}$ Interno $7^{\circ}$ año de Medicina, Facultad de Medicina Universidad de Chile.
}

\begin{abstract}
Cellular and tissue microencapsulation for cellular therapy

Microencapsulation is a technique that protects viable cells in semi-permeable membranes, which allow passage of essential molecules while stopping larger molecules, such as antibodies, involved in the death of transplanted cells. This allows the avoidance of immunosuppressive drugs. Several substances have been used for this purpose, and alginate is one of the most studied and validated. Alginate is extracted from algae present in African and Chilean coasts; different algae can be mixed in variable proportions to produce alginate with distinct characteristics. Commercial alginate evokes an inflammatory response that results in the death of transplanted cells. High purity alginate has already been developed to avoid this issue. There are several applications to this technique, as there are a large number of pathologies that result from the destruction or extraction of tissues, with the consequent loss of function (diabetes mellitus or post-surgical hypoparathyroidism, for example). Finally, there is an additional interest in alginate microencapsulation in this country, given that it can be easily obtained from national algae.
\end{abstract}

Key words: Alginate, microencapsulation, immunosuppressive drugs, cells transplant.

\section{Resumen}

La microencapsulación es una técnica que protege células viables en membranas semi-permeables, que permite el paso de moléculas que son esenciales para la célula y a la vez impide el paso de otras, como los anticuerpos, involucradas en la destrucción celular. Esto permite evitar el uso de drogas inmunosupresoras. Variadas biomacromoléculas pueden ser utilizadas con este fin, siendo el alginato uno de los biopolímeros más validados y estudiados. El alginato es un polisacárido aniónico formado por unidades ácidas b-L-manurónicas y a-L-gulorónicas, extraído desde algas presentes en costas africanas y chilenas. Así, diferentes algas pueden ser mezcladas en proporciones variables para producir alginatos de distintas características. El alginato comercial produce una respuesta inflamatoria que causa la muerte de las células trasplantadas; ya se han desarrollado alginatos de alta pureza para evitar este problema. Existen varias aplicaciones para esta técnica, ya que existen diversas patologías caracterizadas por una pérdida de función por destrucción o extracción de tejido (por ejemplo, diabetes mellitus o hipoparatiroidismo post-quirúrgico). Esta técnica representa un especial interés en Chile, ya que el alginato puede ser obtenido fácilmente a partir de algas chilenas.

Palabras clave: Alginatos, microencapsulación, inmunosupresión, trasplante celular.

*Recibido el 18 de Mayo de 2010 y aceptado para publicación el 23 de Julio de 2010.

Correspondencia: Dr. Patricio Cabané T.

Santos Dumont 999, Santiago, Chile.

patriciocabane@gmail.com 


\section{Introducción}

La microencapsulación busca contener y proteger células viables en membranas semipermeables. Estas membranas deben permitir el paso de moléculas esenciales para la sobrevida celular, y evitar el paso de moléculas más grandes que el tamaño deseado (anticuerpos, complemento). Con esto, se logra crear una barrera inmunológica sin usar drogas inmunosupresoras que conllevan variadas complicaciones derivadas de su uso ${ }^{1}$. Esta técnica, no se usa solamente con el fin de trasplantar células y tejidos, sino también en la producción de moléculas a gran escala y otros tipos de cultivo para experimentación (selección clonal, crecimiento celular con contacto célula a célula, diferenciación celular, etc $)^{2,3}$.

Entre estos dos objetivos principales (viabilidad e inmunoprotección), la microencapsulación requiere múltiples y variados estudios, donde se deben considerar no sólo las características físico-químicas de resistencia y estabilidad del biopolímero a utilizar, sino también los estudios de biocompatibilidad, viabilidad y función celular, e inmunocompetencia. Con este fin se han utilizado varios polímeros naturales y sintéticos para lograr la microencapsulación (alginato, agarosa, quitosano, etc). La presencia de grupos aniónicos en el polímero (carboxílico, sulfónico u otros) interactúan electrostáticamente con soluciones catiónicas formando complejos inter-poliméricos que permiten atrapar células y/o tejidos ${ }^{1,4}$. Actualmente, uno de los biopolímeros naturales más investigados en estudios de microencapsulación corresponde al alginato, el cual es extraído de algas marinas provenientes de costas africanas y chilenas, particularmente de Coquimbo.

\footnotetext{
Alginato

El microambiente y la historia ecológica de las algas es vital para el éxito de la obtención de alginato útil para la microencapsulación. Así es como Edding y cols, reportaron el fracaso en la utilización de alginato para microencapsulación de 20 sitios distintos, principalmente África ${ }^{5}$. Las algas con características óptimas para la recuperación de alginato son Lessonia nigrescens y Lessonia trabeculata, que crecen específicamente en costas chilenas. Estas dos variedades de algas tienen ubicaciones geográficas y condiciones ambientales externas distintas. Mientras L. nigrescens crece en la superficie resistiendo vientos y mareas, $L$. trabeculata crece a 5-30 metros de profundidad. Esto hace que tengan características de elasticidad diferente, lo que se traduce en una constitución molecular distinta de ambas unidades co-monoméricas en el alginato. Así, Lessonia nigre-
}

scens contiene un $60 \%$ de ácido manurónico siendo más elástica, mientras $L$. trabeculata es más rígida y presenta $90 \%$ de ácido gulorónico ${ }^{6-8}$.

El grupo de Zimmermann y cols, tiene la mayor experiencia mundial en el uso de alginato purificado sin filtración, y microencapsulación. Ellos reportaron que una proporción 1:1 (peso:peso) de ambas algas, para la obtención de alginato, permite recuperar concentraciones de ácido manurónico 35\% y gulorónico $65 \%$, otorgándole al alginato propiedades elásticas y mecánicas ideales para la microencapsulación. Es así como este grupo ha desarrollado una técnica sencilla de microencapsulación y de bajo costo para la obtención y purificación de alginato de alta viscosidad para uso clínico (UHV alginate $0,1 \%)^{9,10}$.

\section{Estabilidad y resistencia: Disolución}

La estabilidad y resistencia mecánica de las microesferas depende de la naturaleza química del alginato y se refiere a la capacidad de mantener la integridad de las microesferas en el microambiente específico al que serán sometidas (injerto en músculo, peritoneo, subcutáneo, etc.). Así como también, a propiedades que permitan la disolución controlada del alginato para la recuperación de las células en este ambiente y así permitir estudios de biología molecular. Esta propiedad intrínseca del alginato es lo que permite su fácil purificación con la utilización de etanol y quelantes de iones divalentes como el calcio.

\section{Permeabilidad}

Se ha estudiado la permeabilidad de las membranas semipermeables de alginato en condiciones in vitro, utilizando moléculas de distintos peso molecular o ensayos de flujo de agua libre ${ }^{10}$. Utilizando soluciones de alginatos en concentraciones de $0,9 \%-2 \%$, se detectaron moléculas permeables de peso molecular hasta $147,8 \mathrm{kDa}^{11}$. Esto es vital al considerar que las inmunoglobulinas tienen pesos moleculares desde 150 a $950 \mathrm{kDa}^{12}$.

Otros autores han utilizado microesferas complejas de múltiples capas de Glicol Quitosano-Alginato. En un estudio de Sakai y cols, se confirmó que microesferas formadas por cuatro capas de ambos biopolímeros impiden el paso de inmunoglobulinas y permiten la difusión libre de glucosa, albúmina e insulina ${ }^{13}$.

\section{Microencapsulación y trasplante celular}

\section{Viabilidad}

La viabilidad de las células microencapsuladas se ha evaluado mediante técnicas de visualización 
por microscopia o espectrofotometría, de células vivas y muertas (calceina/propidio, JC1, MTT, etc.), observando mayor viabilidad en los casos de células microencapsuladas en alginato purificado comparado con comercial ${ }^{14}$. Recientemente Moskalenko $\mathrm{y}$ cols, reportaron que la microencapsulación de células o clusters (acúmulos) celulares de paratiroides humana es mejor que la microencapsulación de tejido, ya que se observó necrosis periférica en esta última condición. Por otra parte, la calidad (homogeneidad y superficie de microesferas) es mucho mayor con células o acúmulos celulares (obtenidos por digestión enzimática con colagenasa II). A su vez, la respuesta al calcio extracelular y la vitalidad fue idéntica en el tejido digerido con o sin microencapsulación $^{15}$. Estas características de homogeneidad y superficie lisa son mejores en microesferas de alginato purificado ${ }^{9}$.

\section{Inmunocompetencia}

La inmunocompetencia depende principalmente de la permeabilidad selectiva que evita el paso de inmunoglobulinas y moléculas del complemento ${ }^{12}$. Así, existen evidencias variadas de la citotoxicidad mediada por respuesta inmune específica que corroboran la inmunoprotección otorgada por las microesferas $^{8,16}$. Sin embargo, hay estudios que indican que las microesferas sólo retardan la respuesta inmune citotóxica del huésped contra el alotrasplante de paratiroides ${ }^{17}$. Esto podría deberse a disolución de las microesferas en el tiempo favorecidas por las condiciones del medio o por respuesta inflamatoria.

Cabe destacar que los distintos autores mencionados realizaron sus experimentos con alginatos de algas distintas y a concentraciones distintas. Por lo tanto, la comparación de los resultados debe ser cuidadosa y debemos continuar el desarrollo y búsqueda de la mejor combinación de biopolímeros y procedimientos de microencapsulación para cada tipo celular que nos interese microencapsular.

\section{Biocompatibilidad}

Se refiere al grado de aceptación del huésped frente a los distintos tipos de microesferas, hecho que se traduce en reacciones de inflamación y fibrosis. Los alginatos comerciales son considerados altamente fibróticos ${ }^{8}$, debido a la presencia de impurezas. Los esfuerzos en purificar el alginato apuntan a disminuir el contenido de compuestos mitogénicos que aumentan la reacción a cuerpo extraño, inflamación y fibrosis.

Esto ha sido evaluado por medición de la activación de la cascada de las MAPK y test de estimulación de linfocitos, evidenciando que los alginatos obtenidos de algas chilenas son menos mitogéni$\cos ^{8,10,18}$. Sin embargo, se observa inflamación y fibrosis con todas las formulaciones poliméricas ensayadas, una parte debida a la inflamación producida por el acto quirúrgico, pero no se puede descartar reacción inmune inespecífica (a cuerpo extraño). Es imposible saber en cuanto tiempo habrá una fibrosis tal que comprometa la vitalidad del tejido o células contenidas en las microesferas.

Es importante señalar que existe muy poca experiencia nacional en estos tipos de estudios de microencapsulación, sin embargo, hemos realizado un alotrasplante de tejido paratiroideo microencapsulado. Este alotrasplante fue realizado con alginato comercial con una duración del injerto reportada de 20 semanas $^{19}$.

\section{Conclusiones}

Uno de los materiales poliméricos más ampliamente estudiados y utilizados en la actualidad para la microencapsulación celular es el alginato, biopolímero proveniente de algas marinas. Esto representa un gran desafio de investigación y una importante línea para el desarrollo en tecnología en Chile, ya que son las especies de algas nativas en el país las que proporcionan una fuente ideal de obtención de alginato para microencapsulación, y en la práctica clínica inmediata, nos permitirá ofrecer una terapia alternativa a muchos pacientes con hipoparatiroidismo postquirúrgico que esperan una solución definitiva a su dolencia (alotrasplante de tejido paratiroideo microencapsulado), así como también en otras patologías como la Diabetes Mellitus, que podrían tener en esta técnica una nueva alternativa terapéutica.

\section{Referencias}

1. Uludag H, de Vos P, Tresco P. Technology of mammalian cell encapsulation. Adv Drug Deliv Rev. 2000;42:29-64.

2. Steinert A, Weber M, Dimmler A, Julius C, Schütze $\mathrm{N}$, Nöth U, et al. Chondrogenic differentiation of mesenchymal progenitor cells encapsulated in ultrahighviscosity alginate. J Orthop Res. 2003;21:1090-7.

3. Weber M, Steinert A, Jork A, Dimmler A, Thürmer F, Schütze N, et al. Formation of cartilage matrix proteins by BMP-transfected murine mesenchymal stem cells encapsulated in a novel class of alginates. Biomaterials 2002;23:2003-13.

4. Lee CH, Wang YJ, Kuo SM, Chang SJ. Microencapsulation of parathyroid tissue with photosensitive poly(Llysine) and short chain alginate-co-MPEG. Artif Organs 2004;28:537-42.

5. Edding M, Fonck E, Machiavello J. Lessonia. En I. Akatsuka (ed.), 1994. Biology of Economic Algae. SPB 
Academic Publishing. The Hague, The Netherlands. pp: 407-46.

6. Vásquez JA, Tala F. Lessonia nigrescens along the Chilean coast: evaluation and repopulation experiments. Final Report. Kelco Co. Merck, San Diego, California. 1992, $78 \mathrm{pp}$.

7. Venegas M, Tala F, Fonck E, Vásquez J. Sporangial sori on stipes of Lessonia nigrescens Bory (Laminariales, Phaeophyta): A high frequency phenomenon in intertidal populations of northern Chile. Botánica Marina 1992;35:573-8.

8. Leinfelder U, Brunnenmeier F, Cramer H, Schiller J, Arnold K, Vásquez JA, et al. A highly sensitive cell assay for validation of purification regimes of alginates. Biomaterials 2003;24:4161-72.

9. Zimmermann H, Wählisch F, Baier C, Westhoff M, Reuss R, Zimmermann D, et al. Physical and biological properties of barium cross-linked alginate membranes. Biomaterials 2007;28:1327-45.

10. Zimmermann U, Thürmer F, Jork A, Weber M, Mimietz $\mathrm{S}$, Hillgärtner $\mathrm{M}$, et al. A novel class of amitogenic alginate microcapsules for long-term immunoisolated transplantation. Ann N Y Acad Sci. 2001;944:199-215.

11. Petruzzo P, Cappai A, Ruiu G, Dessy E, Rescigno A, Brotzu G. Development of biocompatible barium alginate microcapsules. Transplant Proc. 1997;29:2129-30.

12. Ouyang W, Chen H, Jones ML, Metz T, Haque T, Martoni $\mathrm{C}$,et al. Artificial cell microcapsule for oral delivery of live bacterial cells for therapy: design, preparation, and in-vitro characterization. J Pharm Pharm Sci. 2004;7:315-24.

13. Sakai S, Ono T, Ijima H, Kawakami K. Control of molecular weight cut-off for immunoisolation by mul- tilayering glycol chitosan-alginate polyion complex on alginate-based microcapsules. J Microencapsul. 2000;17:691-9.

14. Schneider S, Feilen P, Cramer H, Hillgärtner M, Brunnenmeier F, Zimmermann H,. Beneficial effects of human serum albumin on stability and functionality of alginate microcapsules fabricated in different ways. J Microencapsul. 2003;20:627-36.

15. Moskalenko V, Ulrichs K, Kerscher A, Blind E, Otto C, Hamelmann W, et al. Preoperative evaluation of microencapsulated human parathyroid tissue aids selection of the optimal bioartificial graft for human parathyroid allotransplantation. Transpl Int. 2007;20:688-96.

16. Okada N, Miyamoto H, Yoshioka T, Sakamoto K, Katsume A, Saito H, et al. Immunological studies of SK2 hybridoma cells microencapsulated with alginate-poly (L) lysine-alginate (APA) membrane following allogeneic transplantation. Biochem Biophys Res Commun. 1997;230:524-7.

17. Bohrer T, Stelter K, Stelter IS, Dietrich C, Sitter H, Hasse Ch. Microencapsulation of Allogeneic Parathyroid Tissue Succeeds by Delaying Immunization of the Host, not by Protecting the Graft Against the Host's Activated Immune System. Transplantationsmedizin 2006;18:3642.

18. Zimmermann U, Cramer H, Jork A, Thurmer F, Zimmermann H, Fuhr G, et al. Microencapsulation-based cell therapy. In: Reed G, Rehm HJ, editors. Biotechnology Weinheim: Wiley-VCH; 2001. p. 547-71.

19. Cabané P, Gac P, Amat J, Pineda P, Rossi R, Caviedes R, et al. Allotransplant of microencapsulated parathyroid tissue in severe postsurgical hypoparathyroidism: a case report. Transplant Proc. 2009;41:3879-83. 